\title{
Evaluation of an inactivated vaccine for nephropathogenic infectious bronchitis virus
}

\author{
A. Balasubramaniam¹, T. R. Gopalakrishnamurthy², S. Sivaseelan ${ }^{3}$, G. A. Balasubramaniam ${ }^{3}$ and J. J ohnson Rajeswar ${ }^{1}$ \\ 1. Department of Veterinary Microbiology, Veterinary College and Research Institute, Namakkal - 637002, Tamil Nadu, \\ India 2. Poultry Disease Diagnosis and Surveillance Laboratory, Namakkal- 637002, Tamil Nadu, India 3. Department of \\ Veterinary Pathology, Veterinary College and Research Institute, Namakkal - 637002, Tamil Nadu, India \\ Corresponding author: A. Balasubramaniam, email: balasubramaniam72@gmail.com, \\ Tel: 04286266491; Fax: +914286266484
}

Received: 10-06-2012, Accepted: 23-07-2012, Published online: 08-01-2013

How to cite this article: Balasubramaniam A, Gopalakrishnamurthy TR, Sivaseelan S, Balasubramaniam GA and Rajeswar JJ (2013) Evaluation of an inactivated vaccine for nephropathogenic infectious bronchitis virus, Vet. World 6(3): 134-138, doi: 10.5455/vetworld.2013.134- 138

\begin{abstract}
Aim: To evaluate an inactivated vaccine for nephropathogenic infectious bronchitis in broiler with special reference to its ability for passing maternal antibodies to broiler chicks.

Materials and Methods: An inactivated vaccine against nephropathogenic infectious bronchitis (NIB), prepared using an isolate obtained from natural outbreak of NIB was administered to broiler parents at the point of lay, leaving the control birds unvaccinated. Eggs laid below the desired weight $(>52 \mathrm{~g}$ ) by vaccinated hens were utilized for yolk serology. Chicks obtained from hens of both group were subjected for serology and challenge with wild type of nephropathogenic IB isolates. Serology of the yolk and serum was carried out using haemagglutination (HI) test and ELISA.

Results: Yolk serology revealed a geometric mean titre of 415.9 and $15188 \pm 768$ in HI test and ELISA respectively on 28 days post vaccination (dpv) as against 16.0 and $1881 \pm 86$ in yolk from unvaccinated hens. The HI test and ELISA indicated that the level of maternal antibody (MAb) in the chicks obtained from vaccinated hens was significantly $(P<0.01)$ higher on seven days of age than that of chicks from unvaccinated hens. However, the level of Mab of the chicks obtained from vaccinated hens decreased to below the level of protection at two weeks of age. Wild isolate and another isolate obtained from different geographical area were used for challenge dividing the chicks from vaccinated and unvaccinated hens equally. Mortality was observed in the challenged chicks from vaccinated (one in heterologus challenge) and unvaccinated (two) hens. Examination of kidney specimens collected from dead chicks revealed mottling and severe congestion grossly and inflammatory, degenerative and necrotic changes microscopically.
\end{abstract}

Conclusion: The partial cross-protection against heterologous challenge and incomplete protection against homologous challenge with wild isolates were noticed.

Keyowrds: infectious bronchitis virus, nephropathogenicity, protection, vaccine

\section{I ntroduction}

In India, infectious bronchitis (IB) is one of the important poultry diseases and only vaccines prepared from Massachusetts strain 41 (M41) is being used. Despite the use of this live attenuated infectious bronchitis virus (IBV) vaccine, it is not uncommon to find the disease problem. The variant strains of IBV that are antigenically different from vaccine strain could be circulating among chickens in India and recently nephropathogenic IB was reported to be present in nephropathogenic IBV in India [1,2] and China [3]. In Southeast Asia, appearance of a new variant of nephropathogenic IBV is also reported [4,5] whereas in Middle East there was variant IBV causing respiratory symptoms [6]. Thus, determining the genotypic or serotypic identity of a field strain is important for selecting an appropriate vaccine. Serotyping requires antisera to all the IBV isolates circulating throughout the world to actually assign a specific serotype to the particular isolate. In the absence of World reference laboratory for IB, this is highly impracticable.
Narrow range of available vaccine strains and minimal cross-protection among serotypes complicate the process of prevention of IB. Furthermore the rate at which alteration of amino acid sequence in S1 subunit of spike glycoprotein occurs is high, leading to emergence of many serotypes of IBV. Despite vaccination with Mass serotype for prevention of IB, high mortality due to nephropathy occurs in broiler chickens and in layer chickens during growing stage.

An inactivated vaccine prepared from the isolate obtained from nephropathogenic IB outbreak was evaluated with respect to its ability to prevent the disease.

\section{Materials and Methods}

Preparation of oil-adjuvant killed vaccine: The isolate Ind/KA/07/6 was utilized for the preparation of oiladjuvant killed vaccine as per the recommendations of FAO (1993) [7] and as follows:

$\begin{array}{ll}\text { Virus } & : 12.5 \text { per cent }(\mathrm{v} / \mathrm{v}) \\ \text { Liquid paraffin } & : 86.0 \text { per cent }(\mathrm{v} / \mathrm{v}) \\ \text { Spam } 80 & : 1.25 \text { per cent }(\mathrm{v} / \mathrm{v}) \\ \text { Tween } 20 & : 0.25 \text { per cent }(\mathrm{v} / \mathrm{v})\end{array}$


The adjuvant components were first mixed thoroughly and then the formalin inactivated virus was added and blended with the adjuvant using blender. The oil-adjuvant inactivated vaccine was injected at the rate of $0.2 \mathrm{ml}$ per bird intramuscularly.

Safety: The safety of the inactivated virus was assessed in ECE as per British Pharmacopoeia-Veterinary (1993) [8]. The inactivated virus without oil-adjuvant were injected into 10 days old ECE and incubated at $37^{\circ} \mathrm{C}$ for 7 days. The eggs with dead embryos after $24 \mathrm{~h}$ of incubation were kept at $4^{\circ} \mathrm{C}$ and the remaining eggs were chilled at $4{ }^{\circ} \mathrm{C}$ after 7 days for $4 \mathrm{~h}$. Then $0.2 \mathrm{ml}$ of pooled allantoic fluid from live and dead embryos separately were inoculated into ECE and incubated for 7 days. Embryos were examined for IBV lesions.

Potency: The efficacy testing of the inactivated vaccine was carried out as per OIE (2004) [9]. The inactivated vaccine was administered to 20 three-week old cockerel chicks of intramuscularly at $0.2 \mathrm{ml}$ per chick. Another 10 three-week old cockerel chicks were left unvaccinated. Blood was collected from all the chicks before administration of vaccine and subsequently at weekly interval for 3 weeks. The serum separated and heat inactivated at $56^{\circ} \mathrm{C}$ for $30 \mathrm{~min}$. The serum samples were stored at $-20^{\circ} \mathrm{C}$ until tested for the level of antibody to IBV.

After 21 days of post vaccination, all the birds in both groups were inoculated oculonasally with $10^{3.0}$ $\mathrm{EID}_{50}$ of field isolate Ind/KA/07/6 per bird. After 14 days, birds were humanely sacrificed to observe gross lesions.

Healthy broiler parent birds before administration of inactivated IBV vaccine were obtained from a private hatchery in Namakkal, Tamil Nadu. Ideal management with ad libitum sanitized water and broiler parent feed were provided to the birds till the completion of trial, which was approved by Institutional Animal Ethical Committee.

Commercially available Newcastle disease killed vaccine was administered subcutaneously at the rate of $0.5 \mathrm{ml}$ per bird to all the birds during $17^{\text {th }}$ week of age. On $19^{\text {th }}$ week of age, six female and one male parent birds were separated to form vaccine group. Inactivated vaccine prepared from field isolate Ind/KA/07/6 was administered at the rate of $0.5 \mathrm{ml}$ per bird subcutaneously to all the birds in vaccine group. Two female and one male parent birds which were not vaccinated against IB and kept as control group.

Hens started laying from 21 weeks of age. Eggs were collected and stored after labeling at $4^{\circ} \mathrm{C}$ for collection of yolk to assess the level of antibody present in the yolk by ELISA (M/s Synbiotic Corp., USA) and HI test. Eggs weighing $52 \mathrm{~g}$ and above were collected separately from vaccinated and unvaccinated hens and set for hatching. Chicks hatched out from respective groups were used for the challenge study. Twenty four chicks from vaccinated hens and 12 chicks from unvaccinated hens were utilized for the trial. Twelve chicks in vaccine group and six chicks were challenged with $10^{4.5} \mathrm{EID}_{50}$ of wild field isolate Ind/KA/07/1 oculonasally on $6^{\text {th }}$ day of age. Twelve chicks in vaccine group and six chicks were challenged with $10^{4.5} \mathrm{EID}_{50}$ of wild field isolate Ind/TN/07/2 on the same day of age. Chicks were observed for abnormalities and death. Sera from all the chicks were obtained on 7, 14, 21 and 28 days of age to assess the level of antibodies to IBV by HI test and ELISA. Kidney samples from two dead chicks and 12 sacrificed chicks on seven and $14 \mathrm{dpc}$ were subjected for virus isolation and histopathology.

Preparation of egg yolk for serological tests: One ml of yolk was mixed 1:1 (v/v) with PBS and then mixed $1: 2(\mathrm{v} / \mathrm{v})$ with chloroform in a labeled test tube. After incubation for one $\mathrm{h}$ at room temperature, tubes were centrifuged at $383 \mathrm{X} \mathrm{g}$ for $20 \mathrm{~min}$. The clear supernatant was for testing by HI and ELISA and was considered to represent a yolk dilution of 1:2 [10].

Histopathology: Kidneys from dead and sacrificed chicks were collected in 10 per cent neutral buffered formalin. The tissues were embedded in paraffin (50$60^{\circ} \mathrm{C}$ ) and sections were cut to $4-6 \mu$ thickness and stained with haematoxylin and eosin for microscopic examination [11].

Statsitical analysis: The GMT and mean $( \pm$ SE) ELISA titres between the groups were subjected for paired ' $t$ ' test and the comparison of the titre was done at 1 per cent level.

\section{Results}

Safety: All the ten embryos in ECE inoculated with the vaccine were found to be alive. Six days after inoculation, the embryos showed normal growth without any type of lesion comparable with the embryos of control ECE.

Efficacy: The GMT of the chicks vaccinated with inactivated Ind/KA/07/6 before vaccination, 7, 14, 21 and $28 \mathrm{dpv}$ was 13.0, 16.0, 39.4, 157.7 and 512.0 respectively which differed significantly at $<0.05$ level from control group as the GMT of the chicks in control group was lower than vaccine group.

Assessment of immune response by $\mathrm{HI}$ test and ELISA: The GMT of the yolk prepared from eggs obtained from vaccine group differed significantly from control group $(P<0.01)$. GMT reached a peak of 415.9 on $28 \mathrm{dpv}$ and decreased thereafter to 104.0 on 42 dpv. Egg yolk prepared on $28 \mathrm{dpv}$ showed 415.9 and $15188 \pm 768$ in HI test and ELISA respectively which was the highest (Table 1).

The level of antibody in parent birds of vaccinated group was ascertained by HI test and ELISA before vaccination, 14 and $21 \mathrm{dpv}$. Similarly, antibody titre was also assessed in control group. It showed an increase in GMT (294.1) and ELISA titre (9921 \pm 614$)$ during $21 \mathrm{dpv}$ which was significantly $(P>0.01)$ higher than that of control group (Table 2).

The HI test and ELISA indicated level of MAb in 
Table-1. Comparison of GMT and mean ELISA titre of the egg yolk prepared from vaccinated and control groups

\begin{tabular}{|c|c|c|c|c|c|c|c|c|c|c|c|c|}
\hline \multirow[t]{2}{*}{ Group } & \multicolumn{12}{|c|}{ Geometric mean titre $(\mathrm{HI})$ and E LISA titre } \\
\hline & \multicolumn{2}{|c|}{$7 \mathrm{dpv}$} & \multicolumn{2}{|c|}{$14 \mathrm{dpv}$} & \multicolumn{2}{|c|}{$21 \mathrm{dpv}$} & \multicolumn{2}{|c|}{$28 \mathrm{dpv}$} & \multicolumn{2}{|c|}{$35 \mathrm{dpv}$} & \multicolumn{2}{|c|}{$42 \mathrm{dpv}$} \\
\hline $\begin{array}{l}\text { Vaccine } \\
\text { Control }\end{array}$ & $\begin{array}{l}55.7^{\mathrm{a}} \\
22.6\end{array}$ & $\begin{array}{l}3151 \pm 291^{*} \\
2737 \pm 214\end{array}$ & $\begin{array}{l}78.8^{\mathrm{a}} \\
22.6\end{array}$ & $\begin{array}{l}3955 \pm 421^{*} \\
2780 \pm 269\end{array}$ & $\begin{array}{l}194.0^{b} \\
16.0\end{array}$ & $\begin{array}{l}7215 \pm 471^{*} \\
1982 \pm 142\end{array}$ & $\begin{array}{l}415.9^{c} \\
16.0\end{array}$ & $\begin{array}{l}15188 \pm 768^{*} \\
1881 \pm 86\end{array}$ & $\begin{array}{l}288.0^{d} \\
22.6\end{array}$ & $\begin{array}{l}9752 \pm 946 * \\
1881 \pm 86\end{array}$ & $\begin{array}{l}104.0^{\mathrm{a}} \\
22.6\end{array}$ & $\begin{array}{l}6894 \pm 694^{*} \\
1881 \pm 86\end{array}$ \\
\hline
\end{tabular}

GMT with different superscripts differ significantly at $\mathrm{P}<0.01$ level on 21 to $35 \mathrm{dpv}$ in the vaccine group

Table-2. Geometric mean HI titre of vaccinated and unvaccinated parent birds before and after vaccination

\begin{tabular}{lllllll}
\hline Group & \multicolumn{5}{c}{ GMT and ELISA titre } \\
\cline { 2 - 6 } & Before vaccination & \multicolumn{2}{c}{ 14 dpv } & \multicolumn{2}{c}{ 21 dpv } \\
\hline Vaccine & 12.1 & $1621 \pm 163$ & 222.9 & $6947 \pm 427$ & 294.1 & $9921 \pm 614$ \\
Control & 13.0 & $1680 \pm 188$ & 17.1 & $1856 \pm 113$ & 17.1 & $1915 \pm 159$ \\
\hline
\end{tabular}

Table-3. Geometric mean HI titre and mean ELISA titre of chicks obtained from vaccinated and unvaccinated hens

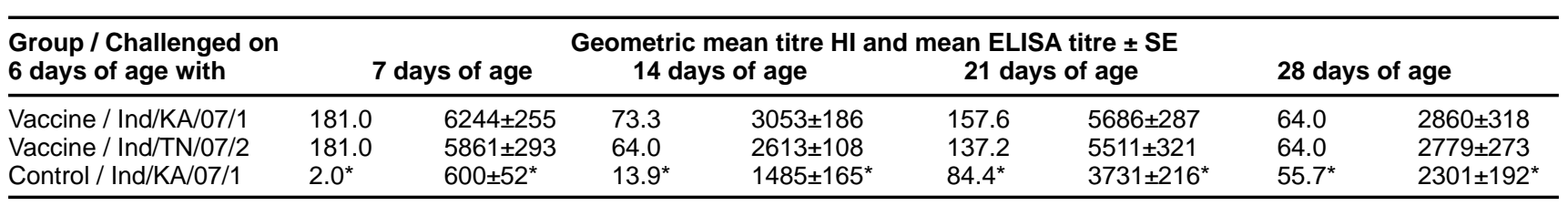

* - Both titres differ significantly $(P<0.01)$ between vaccinated and control groups on 7,14 and 21 days of age

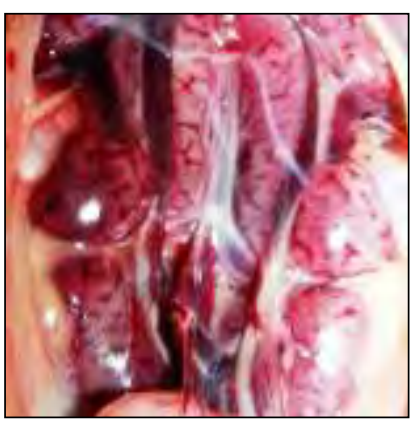

Figure-1. Kidney showing mottling and severe congestion in chick of vaccine group $14 \mathrm{dpc}$.

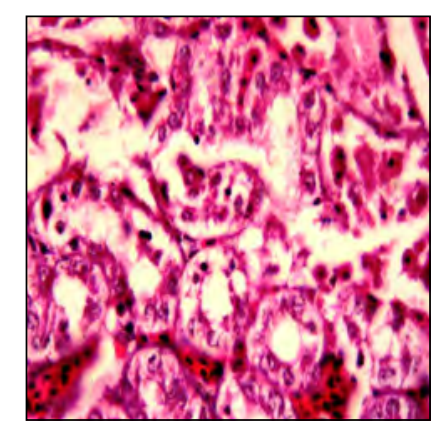

Figure-2. Kidney exhibiting necrosis of tubular epithelial cells, interstitial infiltration of lymphocytes and plasma cells from chick died on nine $d p c$ in the control group (H\&E 1000X) the vaccine group was significantly $(P<0.01)$ higher on seven days of age than the titre observed in control group. However, the MAb level in vaccine group decreased to below the level of protection during second week of age. Chicks in vaccine group challenged with two isolates showed sharp fall in MAb titre during second week and increase in antibody to IBV during third week of age in both the groups. There is significant difference $(P<0.01)$ noticed in GMT and mean ELISA titre on 7, 14, 21 and 28 days of age between the vaccinated and control groups. Increase in antibody titre was also evident in the control group during third week of age (Table 3 ).

Protection study: On $6^{\text {th }}$ day of age, 12 chicks obtained from vaccinated hens and six chicks from unvaccinated hens were challenged with wild isolate Ind/KA/07 and 12 chicks of vaccinated hens and six chicks of unvaccinated hens were challenged with wild isolate Ind/TN/07/2. Two chicks on $9 \mathrm{dpc}$ with isolate Ind/KA/07/1 and another on $13 \mathrm{dpc}$ with isolate Ind/TN/07/2 died in control group. One chick from vaccine group died due to nephropathy on $13 \mathrm{dpc}$ with Ind/TN/07/2. No other clinical signs or mortality was noticed in rest of the chicks of all the groups till the

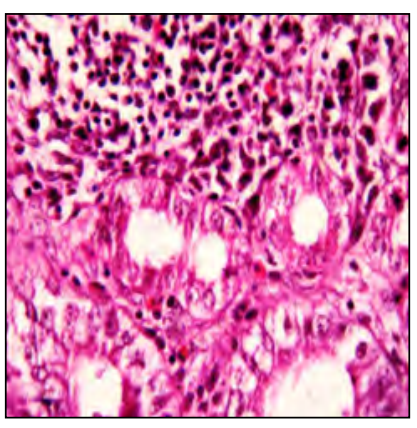

Figure-3. Kidney showing severe necrosis of tubular epithelial cells and congestion in the interstitium from the chick died $13 \mathrm{dpc}$ in control group (H\&E 1000X)

completion of trial.

Pathology of kidney: Grossly, mottling of kidney and nephritis were observed in three dead chicks of control group (9 and $13 \mathrm{dpc}$ ) and mottling and severe congestion of kidney (Fig. 1) in a dead chick of vaccine group $(13 \mathrm{dpc})$. Two chicks each from all the groups on $14 \mathrm{dpc}$ were sacrificed to recover the kidney samples for virus isolation and histopathology. Kidney specimens from dead and sacrificed chicks were subjected for virus isolation in ECE. On third passage, dwarfing and curling of toes of embryo noticed in ECE inoculated with kidney samples from the dead chicks. All other kidney samples including those of control group did not produce embryo lesions in ECE.

Microscopically, inflammatory, degenerative and necrotic changes were observed in kidney from dead chicks. Necrosis of tubular epithelial cells, infiltration of lymphocytes and plasma cells in the interstitium were seen in kidney specimen of chick died on $9 \mathrm{dpc}$ in the control group (Fig. 2). In the kidney specimen from the chick died on $13 \mathrm{dpc}$ in control group, severe necrosis of tubular epithelial cells and accumulation of $\mathrm{RBC}$ in the interstitium were noticed (Fig.3). The kidney from the chick died on $13 \mathrm{dpc}$ in vaccine group 
exhibited haemorrhage in between tubules, degeneration of tubular epithelial cells and accumulation of degenerated cells within the tubules. In the kidney sections recovered from control group on $14 \mathrm{dpc}$ hydrophic degeneration of tubular epithelial cells (Fig. 4) was observed.

\section{Discussion}

Satisfactory results of efficacy trial of the inactivated vaccine indicate that the vaccine is quite enough to induce protective immune response. Nevertheless, the results might not be true indicator as the challenge was carried out with the isolates which may not be virulent enough.

Earlier workers suggested vaccination programmes against IB are based on determination of IBV strains causing the disease in the field and best protection against challenge is achieved by a vaccine containing the homologous strain and the one containing heterologous strains may not induce enough crossprotection [12].

In the present study, GMT differed significantly on 21, 28 and $35 \mathrm{dpv}$ whereas ELISA titre differed significantly on $7,14,21,28$ and $35 \mathrm{dpv}$ in yolk serology between the vaccine and control hens. Comparable results in both the tests show that HI test alone is sufficient for checking the immune response since elevated titre was evident only after $21 \mathrm{dpv}$ in $\mathrm{HI}$ test. Serology of these hens also revealed that the vaccine prepared is able to induce high level of immune response as the titres of unvaccinated hens was significantly lower $(P<0.01)$. This observation correlates with the findings of previous work [13] where HI test detected antibodies between one and two week post infection. However, Gelb and Killian (1987) [14] found that although HI test was serotype-specific when used to detect antibodies after single inoculation with IBV, specificity of the test lower than that of virus neutralization test (VNT).

The immune response in the chicks from vaccinated hens was better than those from unvaccinated hens as assessed by HI test and ELISA on one week of age. However, the MAb level in vaccine group decreased to below the level of protection during second week of age.

Chicks in vaccine group challenged with isolates showed sharp fall in MAb titre during second week and increase in antibody to IBV during third week of age in both the groups. Increase in antibody titre was also evident in the control group during third week of age. The rise in antibody titre might be attributed to response of the chicks to the infection caused by isolate used for challenge.

It was reported that a high level of virus neutralising antibodies against D388 in breeders correlated with higher level of protection in progeny against early challenge. The local protection at 10 days of age was not much higher than in unvaccinated progeny. This indicated that a (heterologous) vaccination at day one does not provide a sufficient protection against challenge with D388 [15].

The broiler chickens with MAb against IBV developed protective immunity by $21 \mathrm{dpv}$ with H120 vaccine administered at one day of age [16] and the same cannot be corroborated with field scenario where there was incidence of nephropathy in broiler chicks even after vaccination with H120 or Mass serotypes. This can be attributed to poor cross-protection and occurrence of infection at very early age. For protection against nephritis, vaccination with homologous attenuated strain in broiler chicks with MAb is necessary [17]. In contrast, the inactivated vaccine developed utilizing nephropathogenic IBV strain was found to protect NIB caused by different variants $[18,19]$.

Cross-protection evinced in this trial can be attributed to the fact that antibodies produced might neutralize the heterologous strain where challenges with four European serotypes in vaccinated and unvaccinated broilers yielded 100 per cent serotypespecific VNT [13]. A live attenuated nephropathogenic IB virus vaccine offered high level of cross-protection for variants [18]. However, especially after challenge of the vaccinated broilers with a heterologous serotype, antibodies were detected against IBV serotypes to which the birds had not been exposed. On the contrary, it was showed that VNT for different serotypes performed on chicken embryo kidney cells, was highly serotype-specific after one inoculation [14].

Death due to nephritis of two chicks inoculated with isolates on 9 and $13 \mathrm{dpc}$ in control group and death of a chick due to nephropathy in vaccine group on 13 dpc with Ind/TN/07/2 indicates that the presence of $\mathrm{MAb}$ in the vaccine group offers protection to the chicks against challenge with the isolate (Ind/KA/07/1) procured from similar location as that of isolate used for inactivated vaccine preparation. There is some level of cross-protection as only one chick died out of 12 chicks inoculated with Ind/TN/07/2 as against two out of 12 in control group.

Mottling of kidney and nephritis observed in the dead chicks of control group denoted that the virus was able to replicate in kidney and caused damage. Similar lesion was observed in dead chick inoculated with Ind/TN/07/2 which indicated that presence of strainspecific antibodies is necessary to prevent nephropathogenicity.

Severe renal lesions such as necrosis of tubular epithelium, infiltration of lymphocytes and plasma cells in the interstitium noticed in control group chicks indicated that great invasiveness of both isolates. Similar finding was reported in SPF chicks [20]. Observation of moderate renal lesions such as haemorrhage in between tubules, degeneration of tubular epithelium and accumulation of degenerated cells within the tubules in a chick of vaccine group died on $13 \mathrm{dpc}$ denoted that nephropathogenicity is minimized. Similar finding was reported where there was penetration and destruction of the tubular epithelium and the invasion of the surrounding interstitium by heterophils 
in IB-MAb positive chicks challenged with Australian Tstrain [21].

In India, live and inactivated Mass type vaccines are extensively used for the control of IB. Further, it is likely that more variants may evolve in future and it is imperative to monitor the prevalence of variants or serotypes continuously. Advantage of IBV vaccination with multiple strains or serotypes which can induce better cross-protection cannot be enjoyed by poultry growers in India as only Mass type vaccine is available. From the findings of the present study, it is concluded that the partial cross-protection against heterologous challenge and incomplete protection against homologous challenge with wild isolates were evident.

\section{Authors' contributions}

$\mathrm{AB}$ conceived the work and executed the plan of work desired by all other authors. SS and GAB carried out histopathological work. TRG and JJR carried out serological work. All authors participated in drafting and revision of manuscript. All authors read and approved the final manuscript.

\section{Acknowledgements}

The authors are thankful to the Tamil Nadu Veterinary and Animal Sciences University and the Dean, Veterinary College and Research Institute, Namakkal - 637002, India for providing the facilities to conduct the trial.

\section{Competing interests}

Authors declare that they have no competing interest.

\section{References}

1. Bayry, J., Goudar, M.S., Nighot, P.K., Kshirsagar, S.G., Ladman, B.S., Gelb Jr., J., Ghalsasi, G.R. and Kolte, G.N. (2005) Emergence of a nephropathogenic avian infectious bronchitis virus with a novel genotype in India. J. Clin. Microbiol., 43: 916-918.

2. Singh, F., Shakya, S. and Rawat, N. (2009) An outbreak of nephropathogenic avian infectious bronchitis in broiler flocks of Chhattisgarh region. Indian Journal of Poultry Science 44:379-381.

3. Bing G.X., Liu, X, Pu, J., Liu, Q.F., Wu, Q.M. and Liu, J.H. (2007) Different genotypes of nephropathogenic infectious bronchitis virus co-circulating in chicken population in China. Virus Genes 35: 333-337.

4. Kang, S.C., Eun, K.L., Woo, J.J., Mi, J.P. Jin, W.K. and Jun, H.K. (2009) Pathogenicity and antigenicity of a new variant of Korean nephropathogenic infectious bronchitis virus. $J$. Vet. Sci., 10:357-359.

5. Pohuang, T. and Sasipreeyajan, J. (2012) The pathogenesis of a new variant genotype and QX like infectious bronchitis virus isolated from chickens in Thailand. Thai J Vet Med., 42: 51-57.
6. Ababneh, M., Dalab, A.E., Alsaad, S. and Al-zghoul, M. (2012) Presence of infectious bronchitis virus strain CK/CH/LDL/97I in the Middle East. Vet Sci., doi: 10.5402/ 2012/201721.

7. FAO. (1993) Manual for the production of Marek's disease, Gumboro disease and inactivated Newcastle disease vaccines. 89: 29 - 46.

8. British Pharmacopoeia (Veterinary). (1993) Avian infectious bronchitis -inactivated vaccine and live attenuated vaccine. HMSO, London, pp. 110-111.

9. O.I.E. (2004) Manual of standards of diagnostic tests and vaccines. Avian infectious bronchitis, 4th edn. pp. 569- 561.

10. Piela, P.H., Gulka, C.M., Yates, V.J. and Chang, P.W. (1984) Use of egg yolk in serological tests (ELISA and HI) to detect antibody to Newcastle Disease, Infectious bronchitis and Mycoplasma gallisepticum. Avian Dis., 28: 877-883.

11. Bancroft, J.D and Stevens, A. (1996) Theory and practice of histological techniques. $4^{\text {th }}$ edn. Churchill Livingstone, London.

12. Gelb, J. Jr., Perkins, B.K., Rosenberger, J.K. and Allen, P.H. (1981) Serologic and cross-protection studies with several infectious bronchitis virus isolates from Delmarva-reared broiler chickens. Avian Dis., 25: 655 - 666.

13. de Wit, J. J., Mekkes, D.R., Kouwenhoven, B. and Verheijden, J.H.M. (1997) Sensitivity and specificity of serological tests for detection of infectious bronchitis virus induced antibodies in broilers. Avian Pathol., 26: 105 - 118.

14. Gelb, J. Jr., and Killian, S.L.(1987) Serum antibody responses of chickens following sequential inoculations with different infectious bronchitis virus serotypes. Avian Dis., 31: $513-522$.

15. de Wit, J.J., Nieuwenhuizen, J. and Fabri, T.H.F. (2006) Protection by maternally derived antibodies and vaccination at day of hatch against early challenge with IBV serotype D388. In U.H. Redmann, and F. Kaleta (Eds.). Proceedings of $\mathrm{V}^{\text {th }}$ International symposium on avian corona- and pneumoviruses and complicating pathogens. Rauischholzhausen, Germany (pp. 313-318).

16. Davelaar, F. G. and Kouwenhoven, B. (1980) Influence of maternal antibodies on vaccination of chicks of different ages against infectious bronchitis. Avian Pathol., 9: 499-510.

17. Pensaert, M and Lambrechts, C. (1994) Vaccination of chickens against a Belgian nephropathogenic strain of infectious bronchitis virus B1648 using attenuated homologous and heterologous strains. Avian Pathol., 23: 631 $-641$.

18. Lim T.H., Kim M.S., Jang J.H., Lee D.H., Park J.K., Youn H.N., Lee J.B., Park S.Y., Choi I.S. and Song C.S. (2012) Live attenuated nephropathogenic infectious bronchitis virus vaccine provides broad cross protection against new variant strains. Poult Sci., 91: 89-94.

19. Xi, Q., Ji, J., Xie, J., Chen, F., Cai, M., , B., Xue, C., Ma, J. and $\mathrm{Bi}$, Y. (2011) Epidemiology and immunoprotection of nephropathogenic avian infectious bronchitis virus in southern China. Virology Journal 8: 484-489.

20. Chen, B.Y., Hosi, S., Nunoya, T. and Itakura, C. (1996) Histopathology and immunohistochemistry of renal lesions due to infectious bronchitis virus in chicks. Avian Pathol., 25: 269-283.

21. Pohl. R. (1974) The histopathogenesis of nephritis nephrosis syndrome. Avian Pathol., 3: 1-13. 